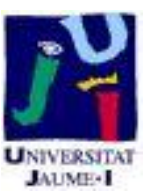

Título artículo / Títol article:

Multi-class determination of personal care products and pharmaceuticals in environmental and wastewater samples by ultra-high performance liquidchromatography-tandem mass spectrometry

Autores / Autors:

Emma Gracia-Lor, Marian Martínez,

Juan V. Sancho, Gustavo Peñuela, Félix Hernández

Revista:

Talanta (2012) Volume 99

Versión / Versió:

Preprint de l'autor

Cita bibliográfica / Cita bibliogràfica (ISO 690):
Emma Gracia-Lor, Marian Martínez, Juan V. Sancho, Gustavo Peñuela, Félix Hernández. Multi-class determination of personal care products and pharmaceuticals in environmental and wastewater samples by ultra-high performance liquidchromatography-tandem mass spectrometry. Talanta, Volume 99, 15 September 2012, Pages 1011-1023 


\title{
Multi-class determination of personal care products and pharmaceuticals in environmental and wastewater samples by ultra-high performance liquid-chromatography-tandem mass spectrometry
}

Talanta, 99 (2012) 1011-1023

\author{
Emma Gracia-Lor ${ }^{\mathrm{a}}$, Marian Martínez ${ }^{\mathrm{b}}$, Juan V. Sancho ${ }^{\mathrm{a}}$, Gustavo Peñuela ${ }^{\mathrm{b}}$ \\ and Félix Hernández ${ }^{a}$ \\ ${ }^{a}$ Research Institute for Pesticides and Water, University Jaume I, Avda. Sos \\ Baynat, E-12071 Castellón, Spain \\ ${ }^{b}$ GDCON-Grupo de Diagnóstico y Control de la Contaminación, Facultad de \\ Ingeniería, Universidad de Antioquia, AA 51922, Medellín, Colombia
}

\begin{abstract}
In this work, a multi-class method for the simultaneous determination of 17 emerging contaminants, including pharmaceuticals and personal care products, has been developed. Target analytes were two anti-inflammatories, a lipid regulator agent, two angiotensin II antagonists, two antiepileptic drugs and a diuretic. Among personal care products, four preservatives and five UV filters were included. The method is based on solid-phase extraction (SPE) using Oasis HLB cartridges followed by ultra-high performance liquid chromatographytandem mass spectrometry (UHPLC-MS/MS). Up to three simultaneous transitions per compound were acquired to assure a reliable identification. A detailed study of the extraction process efficiency and matrix effects was carried out in surface water and effluent wastewater. The use of isotope-labeled internal standards (ILIS) was tested to compensate both potential SPE losses during sample extraction and signal suppression/enhancement observed, especially in EWW. Satisfactory correction in all water samples was only ensured when the own analyte ILIS was used. The use of analogues ILIS was a rather useful approach for correction in the majority of the samples tested when analyte ILIS was unavailable. The method was successfully validated in five different surface water (SW) samples and five effluent wastewater (EWW) samples spiked at two concentration levels $(0.05$ and $0.5 \mu \mathrm{g} / \mathrm{L}$ in SW; 0.1 and $0.5 \mu \mathrm{g} / \mathrm{L}$ in EWW). The developed method was applied to the analysis of 22 samples (SW and EWW) from the Spanish Mediterranean area and 51 reservoir water samples from Colombia. Personal care products were frequently detected, with the highest concentrations corresponding to benzophenone and benzophenone-4 (samples from Spain), and methylparaben (samples from Colombia). Several pharmaceuticals were detected in the Spanish samples, where irbesartan
\end{abstract}


and valsartan - two Angiotensin II antagonists that are not commonly monitored in the aquatic environment - were the compounds most frequently detected.

\section{Keywords}

Pharmaceuticals; Personal care products; Ultra-high performance liquid chromatography; Tandem mass spectrometry; Matrix effects; Surface water and wastewater.

\section{Introduction}

Pharmaceuticals and personal care products (PPCPs) are a diverse group of chemicals that include human and veterinary drugs, food supplements and other chemicals used in cosmetics, fragrances and sun-screen agents [1]. They have become the focus of global environmental researchers' attention over the last decade [2] due to their extensive human and veterinary consumption and their potential negative impact on the environment, living organisms and human health. PPCPs are suspected to cause elevated rates of cancer, reproductive impairment in humans and other animals and development and spread of antimicrobial resistance [2-4]. Recent studies reported that these contaminants do not need to be persistent in the environment to cause negative effects due to their continuous release $[1,5,6]$. They have been detected in different natural water systems that include rivers, lakes and reservoirs [7-9].

PPCPs enter into the aquatic environment mainly through effluents of wastewater treatment plants (WWTPs) [6]. A few compounds are transformed or retained in the sludge by conventional water treatment processes. However, most of compounds are persistent and polar, and survive the passage through WWTPs, being released via effluents into receiving aquatic environments [1-3].

Pharmaceuticals have been extensively studied in the last years due to their large consumption and environmental implications. However, until recently, less interest has been paid to the presence of personal care products (PCPs) in environmental waters [10]. PCPs include UV-filters, preservatives, antimicrobials, musk fragrances, insect repellents and, in general, ingredients or excipients used in cosmetics, food supplements, shampoos, toothpastes, sun screens agents, antiseptics and personal care products formulations and manufacture [1, 10]. Among them, UV filters and preservatives are considered of higher relevance. UV filters are used as ingredients in some sunscreens and a wide variety of cosmetics (skin and hair care products, lotions, creams, fragrances) [11-13]. These compounds enter the aquatic environment either directly via wash-off from skin and cloth during recreational activities, or indirectly via wastewater or swimming pool waters [11]. The most commonly UV filters are benzophenones. Two of them, benzophenone- 3 and benzophenone- 4 are included in the list of compounds that can be employed as UV filters in sunscreen cosmetic products according to the European Union Legislation [14]. Benzophenone, benzophenone-1 and benzophenone-2 seem to present hormonal activity [11-12]. Moreover, benzophenone has been 
listed among chemicals suspected of having endocrine disrupting effects [12]. These compounds are characterised by the presence of aromatic rings, often with attached hydrophobic groups. They are lipophilic and can therefore be accumulated in the human body and the environment [15].

Among the group of preservatives, parabens are the most commonly used. These are esters of p-hydroxybenzoic acid and are suspected to be endocrine disruptors $[10,16]$ Parabens are widely used, due to their anti-bactericidal and anti-fungicidal properties, in cosmetic products and pharmaceuticals, and in food and beverage processing [16-18].

Advanced analytical methodology is needed in order to study the occurrence and distribution of PPCPs in the environment. Most of recent methods are based on liquid chromatography-tandem mass spectrometry (LC-MS/MS) using triple quadrupole (QqQ) analyzer [10], which has become the technique of choice in this field due to its high selectivity and sensitivity [2]. However, one of the major drawbacks associated to LC-MS/MS methods is matrix effect, which results in suppression or enhancement of the analyte signal due to the presence of co-extracted matrix components that affect analyte ionization. These undesirable effects can hamper the identification, and drastically affect the quantification of analytes, especially when dealing with complex matrices [19].

The goal of this paper is to develop rapid, accurate and sensitive analytical methodology based on UHPLC-MS/MS for the simultaneous determination (quantification and confirmation) of PPCPs in surface and wastewater. A notable number of PPCPs (17 compounds) has been included in the method scope in order to have wider and more realistic knowledge on the presence of these compounds in the environment. The main benzophenones, including those listed in the EU legislation, and the parabens most extensively used in cosmetics and food processing, methyl-, ethyl- and buthyl-paraben [10] have been included in the target list of analytes. Several ILIS have been tested for correction of matrix effects and/or potential losses associated to the SPE step. The method was applied to the analysis of 73 samples (surface water, effluent wastewater and reservoir water) collected at different sites in Spain and Colombia. Although emerging contaminants are still unregulated in water, their presence is a matter of concern, mainly in those cases where treated water is used for drinking water supply. This occurs in Colombia, where artificial reservoirs are frequently used to this aim. Reservoirs are supplied with water from rivers or tributaries, which receive urban and agricultural wastewater discharges. For this reason, it is important to monitor water quality in Colombia's reservoirs, specifically for those PPCPs used in human applications and households. The presence and distribution of PPCPs in Colombia have not been reported, and to the best of our knowledge, this is the first study reporting data on PPCPs in this country. The methodology developed in this work will be implemented in the laboratory of GDCON group from Antioquia University (Colombia) since 2012. 


\section{Experimental}

\subsection{Reagents and chemicals}

Reference standards were purchased from Sigma-Aldrich (St Louis, MO, USA), LGC Promochem (London, UK), Dr. Ehrenstorfer (Augsburg, Germany) and Toronto Research Chemicals (Ontario, Canada). Their chemical structure is shown in Fig. 1.

Isotopically labeled compounds diclofenac- $\mathrm{d}_{4}$, ibuprofen- $\mathrm{d}_{3}$, valsartan- $\mathrm{d}_{8}$, ethylparaben$d_{4}$ and benzophenone-3- $d_{5}$, were from CDN Isotopes (Quebec, Canada).

HPLC-grade methanol $(\mathrm{MeOH})$ and HPLC-grade acetonitrile $(\mathrm{ACN})$ were purchased from Scharlab (Barcelona, Spain). HPLC-grade water was obtained from distilled water passed through a Milli-Q water purification system (Millipore, Bedford, MA, USA). Formic acid $(\mathrm{HCOOH}$, content $>98 \%)$, ammonium acetate $\left(\mathrm{NH}_{4} \mathrm{Ac}\right.$, reagent grade) and ammonia $\left(\mathrm{NH}_{3}\right.$, solution $32 \%$, reagent grade) were supplied by Scharlab (Barcelona, Spain).

Individual stock solutions of PPCPs were prepared dissolving $25 \mathrm{mg}$, accurately weighted, in $50 \mathrm{~mL}$ methanol, obtaining a final concentration of $500 \mathrm{mg} / \mathrm{L}$. Intermediate solutions were prepared in methanol, and were used to prepare a mix of all compounds at 500 $\mu \mathrm{g} / \mathrm{L}$ in methanol-water $(40: 60, \mathrm{v} / \mathrm{v})$. This mix solution was subsequently diluted with HPLCgrade water to prepare working solutions.

Individual stock solutions of ILIS were prepared in methanol. A mix working solution at $250 \mu \mathrm{g} / \mathrm{L}$ was prepared in water and used as surrogate.

All standard solutions were stored in amber glass bottles. Stock solutions were stored at $-20^{\circ} \mathrm{C}$, and intermediate and working solutions at $4{ }^{\circ} \mathrm{C}$.

Cartridges used for SPE were Oasis HLB $(60 \mathrm{mg})$ and Oasis MCX (150 mg) from Waters (Milford, MA, USA). 


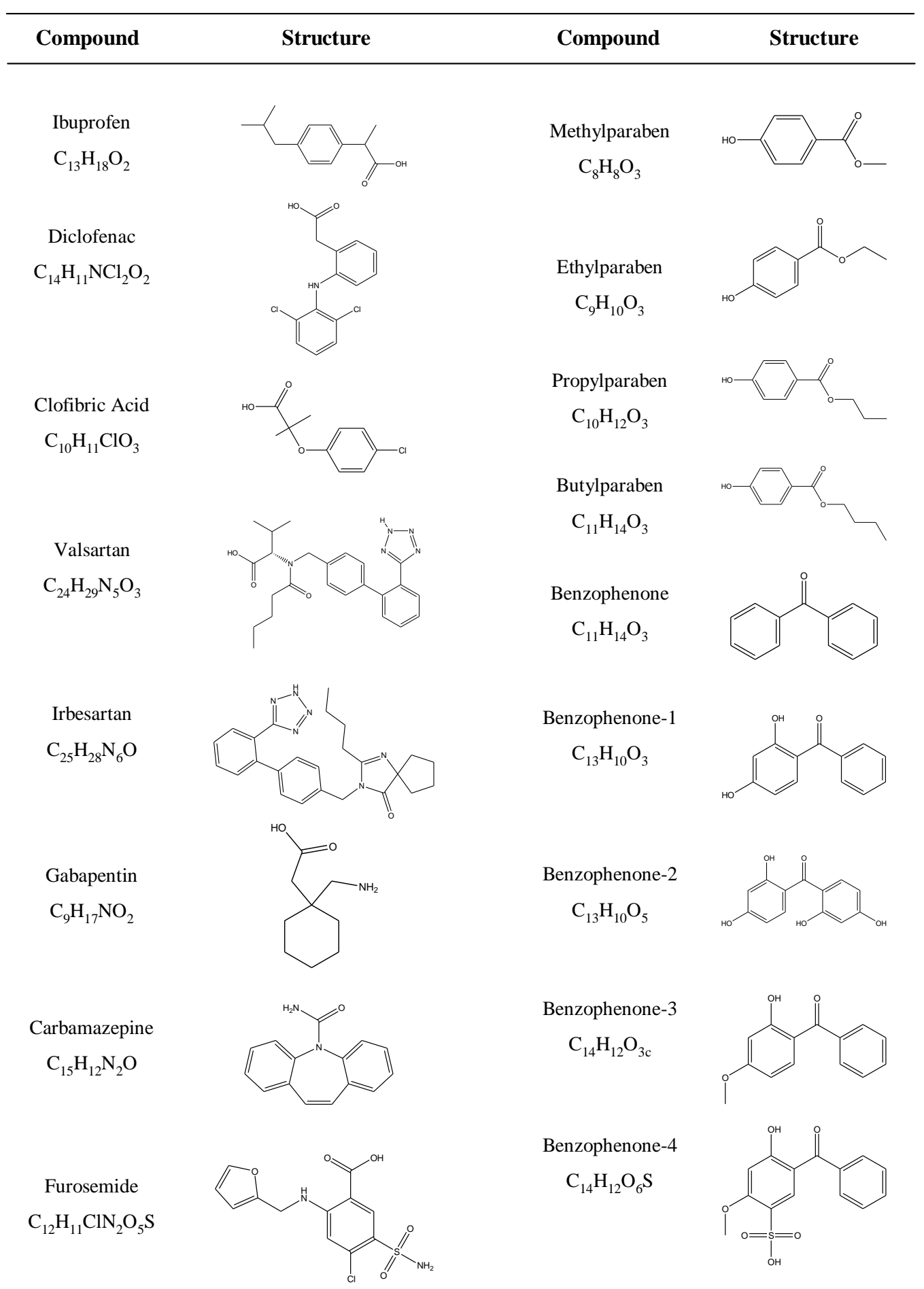

Fig. 1. Structures and molecular formula of the selected compounds. 


\subsection{Liquid chromatography}

UHPLC analysis were carried out using an Acquity UPLC system (Waters Corp., Milford, MA, USA), equipped with a binary solvent manager and a sample manager. Chromatographic separation was performed using an Acquity UPLC BEH C18 column, $1.7 \mu \mathrm{m}$, $50 \mathrm{~mm} \times 2.1 \mathrm{~mm}$ (i.d.) (Waters) at a flow rate of $0.3 \mathrm{~mL} / \mathrm{min}$. The column was kept at $40{ }^{\circ} \mathrm{C}$ and the sample manager was maintained at $5{ }^{\circ} \mathrm{C}$. Mobile phase consisted of a water $0.01 \%$ $\mathrm{HCOOH} /$ methanol gradient. The methanol percentage was changed linearly as follows: 0 min, 10\%; $9 \mathrm{~min}, 90 \%$; $10 \mathrm{~min}, 90 \% ; 10.1 \mathrm{~min} ; 10 \%$. Analysis run time was $12 \mathrm{~min}$. The sample injection volume was $20 \mu \mathrm{L}$.

\subsection{Mass spectrometry}

A TQD (triple quadrupole) mass spectrometer with an orthogonal Z-spray-electrospray (ESI) was used (Waters Corp., Milford, MA, USA). Drying gas as well as nebulising gas was nitrogen generated from pressurized air in a $\mathrm{N}_{2}$ LC-MS (Claind, Teknokroma, Barcelona, Spain). The cone gas and the desolvation gas flows were set at 60 and $1200 \mathrm{~L} / \mathrm{h}$, respectively. For operation in MS/MS mode, collision gas was Argon 99.995\% (Praxair, Valencia, Spain) at $2 \times 10^{-3} \mathrm{mbar}$ in the T-Wave collision cell. Capillary voltages of $-3.0 \mathrm{kV}$ (negative ionization mode) and $3.5 \mathrm{kV}$ (positive ionization mode) were applied. The interface temperature was set to $500{ }^{\circ} \mathrm{C}$ and the source temperature to $120^{\circ} \mathrm{C}$. A dwell time of $0.01 \mathrm{~s}$ was selected.

Masslynx 4.1 (Micromass, Manchester, UK) software was used to process quantitative data.

\subsection{Recommended procedure}

Water samples were centrifuged at $4500 \mathrm{rpm}$ for $5 \mathrm{~min}$, when suspended particulate matter was observed. $100 \mathrm{~mL}$ water sample were taken and $100 \mu \mathrm{L}$ of the ILIS mix working solution were added to give a final concentration for each ILIS of $0.25 \mu \mathrm{g} / \mathrm{L}$. Oasis HLB cartridges were conditioned with $3 \mathrm{~mL} \mathrm{MeOH}$ and $3 \mathrm{~mL}$ HPLC-grade water before use. Then, the samples were loaded onto the cartridge and, after drying under vacuum, analytes were eluted with $5 \mathrm{~mL}$ methanol. The extract was evaporated to dryness under a gentle nitrogen stream at $40{ }^{\circ} \mathrm{C}$ and reconstituted with $1 \mathrm{~mL} \mathrm{MeOH}$-water (10:90, v/v). Finally, $20 \mu \mathrm{L}$ were injected in the UHPLC-MS/MS system under the conditions shown in Table 1. Quantification was made with calibration standards prepared in solvent, using relative responses analyte/ILIS, or absolute responses, depending on whether ILIS was used for correction or not. ILIS were used to correct for matrix effects and/or SPE potential errors as shown in Tables 2 and 3. 


\subsection{Validation study}

The linearity of the method was studied by analyzing standard solutions (in triplicate) at seven concentrations in the range from 1 to $100 \mu \mathrm{g} / \mathrm{L}$ (equivalent to $0.01-1 \mu \mathrm{g} / \mathrm{L}$ in the water sample). Satisfactory linearity using least squares regression was assumed when the correlation coefficient $(r)$ was higher than 0.99 and residuals lower than $30 \%$ without significant trend, based on relative responses (analyte peak area/ILIS peak area), except for those compounds that were quantified without ILIS (absolute response).

Accuracy (expressed as percentage recovery) and precision (expressed in terms of relative standard deviation (RSD)) were studied by means of recovery experiments in several surface water (SW) and effluent wastewater (EWW) spiked at two concentrations (0.05 in 0.5 $\mu \mathrm{g} / \mathrm{L}$ in SW; 0.1 and $0.5 \mu \mathrm{g} / \mathrm{L}$ in EWW). The method was validated in five SW samples collected in different sites of the Mediterranean Spanish area of Valencia (Mijares and Jucar rivers, and Albufera de Valencia, Clot de Burriana, and Tavernes de la Valldigna lakes) and five 24-h composite EWW samples collected from the Castellón de la Plana WWTP along five consecutive days. For each individual sample, recovery experiments were performed in duplicate, giving a total number of 10 data for SW and 10 for EWW at each spiked concentration.

The limit of quantification (LOQ) was estimated for a signal-to-noise $(S / N)$ ratio 10 from the sample chromatograms at the lowest validation level tested, using the quantification transition. True blank samples were not found for several analytes, which were present in the samples tested. In these cases, LOQ values were estimated from the analyte levels quantified in the non-spiked "blanks". The instrumental limit of detection (LOD) was estimated for $S / N=3$ from the chromatograms of standards at the lowest concentration level.

\subsection{Application to water samples}

The method was applied to 73 samples (surface water, effluent wastewater and reservoir water) collected at different sites in Spain and Colombia.

Eleven SW samples were collected at selected sites from the Spanish Mediterranean area of Valencia. Another eleven EWW were also collected, consisting on 24-h composite urban wastewater samples, from different WWTPs of the same area. All samples were stored in the dark at $-18{ }^{\circ} \mathrm{C}$ in polyethylene high-density bottles until analysis.

Fifty-one reservoir water samples were collected from two reservoirs situated in the department of Antioquia (Colombia) that are used for drinking water supply. In total, eleven sampling locations were selected for reservoir 1 (located in the east of Antioquia), and 10 for reservoir 2 (in the north of Antioquia) and collected at three depths in the water column: subsurface, photic zone limit and reservoir bottom. Due to the low depth, it was only possible to 
take samples at the surface and at the bottom in some stations. Samples were collected in July and August 2011 using a Schindler bottle. Then, $100 \mathrm{~mL}$ of each sample were transferred to a polyethylene bottle. A total of 51 samples were collected and transported to the laboratory under cooled conditions $\left(4^{\circ} \mathrm{C}\right)$. Upon reception in the Colombian laboratory, samples were immediately processed (SPE) and the cartridges were sent to Spain for LC-MS/MS analysis. No stability test of analytes on the SPE cartridges was made.

\section{Results and discussion}

In this work, 17 pharmaceuticals and personal care products (PPCPs) were studied. Pharmaceuticals selected belong to different therapeutical groups: anti-inflammatories, antiepileptic drugs, lipid regulators, angiotensin II antagonists and diuretics. The choice of diclofenac, ibuprofen and clofibric acid was based on their high usage in both Spain and Colombia [20]. Valsartan, irbesartan, gabapentin, carbamazepine and furosemide have been frequently detected in wastewater of the Valencian area [21]. The remaining nine compounds were personal care products (UV filters and preservatives). They were selected due to their wide use and to their potential harmful effects on human and aquatic organisms [10, 22].

\subsection{MS and MS/MS optimization}

Full-scan and MS/MS mass spectra of analytes were obtained from infusion of $1 \mathrm{mg} / \mathrm{L}$ methanol/water (50:50, v/v) individual standard solutions at a flow rate of $10 \mu \mathrm{L} / \mathrm{min}$. For those compounds that showed abundant sodium adduct $[\mathrm{M}+\mathrm{Na}]^{+}$(e.g. carbamazepine), the addition of $\mathrm{HCOOH}$ into the infusion vial favored the $[\mathrm{M}+\mathrm{H}]^{+}$formation. $\mathrm{NH}_{4} \mathrm{Ac}$ was also added into the infusion vial in order to evaluate the possible presence of ammonium adducts, or to minimize the $[\mathrm{M}+\mathrm{Na}]^{+}$formation. Although sodium adducts decreased in presence of ammonium, the abundance of the protonated molecules was lower than using formic acid. Therefore, formic acid was selected as additive.

Valsartan, irbesartan, benzophenone-1, benzophenone-2 and benzophenone-3 were ionized in both positive and negative modes. With the exception of benzophenone-2, positive ionization mode was selected because of the better sensitivity reached under this mode. The most abundant ion was $[\mathrm{M}+\mathrm{H}]^{+}$, or $[\mathrm{M}-\mathrm{H}]^{-}$, and it was selected as precursor ion. The presence of halogenated atoms $(\mathrm{Cl})$ in some compounds (diclofenac and clofibric acid) allowed using two different precursor ions (corresponding to ${ }^{35} \mathrm{Cl}$ and ${ }^{37} \mathrm{Cl}$, respectively).

Three SRM transitions were selected for each compound to assure the reliable confirmation of the compound detected. The most abundant product ion was used for quantification $(Q)$ whereas the second and the third most sensitive transitions were used for confirmation $\left(q_{1}\right.$ and $\left.q_{2}\right)$. For ibuprofen, diclofenac, irbesartan, parabens and some 
benzophenones, only one (ibuprofen) or two transitions (the rest of compounds) could be monitored due to their poor fragmentation. For example, for parabens the $m / z=92$ product ion, corresponding to $\left[\mathrm{C}_{6} \mathrm{H}_{4} \mathrm{O}\right]^{-}$, was selected. This ion was the most abundant fragment for methylparaben, ethylparaben and propylparaben, and therefore it was selected for quantification, while for butylparaben it was selected for confirmation. For this compound, [M$\left.\mathrm{H}-\mathrm{CH}_{2} \mathrm{CH}_{2} \mathrm{CH}_{2} \mathrm{CH}_{3}\right]^{-}$, corresponding to the lost of a butyl radical, was the most abundant ion $[22,23]$.

For benzophenone-1 and benzophenone-3, similar fragment ions were obtained due to their analogous chemical structure. In both cases, the loss of the benzene group $\left[\mathrm{M}+\mathrm{H}-\mathrm{C}_{6} \mathrm{H}_{6}\right]^{+}$ corresponded to the main product ion (selected for quantification), while $\left[\mathrm{C}_{7} \mathrm{H}_{5} \mathrm{O}\right]^{+}$was selected for confirmation, as previously reported [24].

Regarding ILIS, only one transition was required. In the case of diclofenac- $\mathrm{d}_{4}$, the transition 300.1>256.1 was chosen in order to avoid the overlap between the natural analyte (isotope peak due to the presence of two chlorine atoms; $2 \mathrm{Cl}^{37}$ ) and the ILIS signal $\left(d_{4}\right)$, which would have occurred if the transition 298.1>254.1 had been chosen. In order to obtain enough points to define the chromatographic peak, the SRM transitions were distributed along six overlapping windows using dwell times of $10 \mathrm{~ms}$. This favorable overlapping between positive and negative time windows was possible due to the low positive-to-negative-switching time (20 ms) attainable by the triple quadrupole analyzer used in this work.

Mass spectrometry parameters, precursor and product ions selected, instrumental LODs and ion ratios $(Q / q)$ used for confirmation are shown in Table 1. 


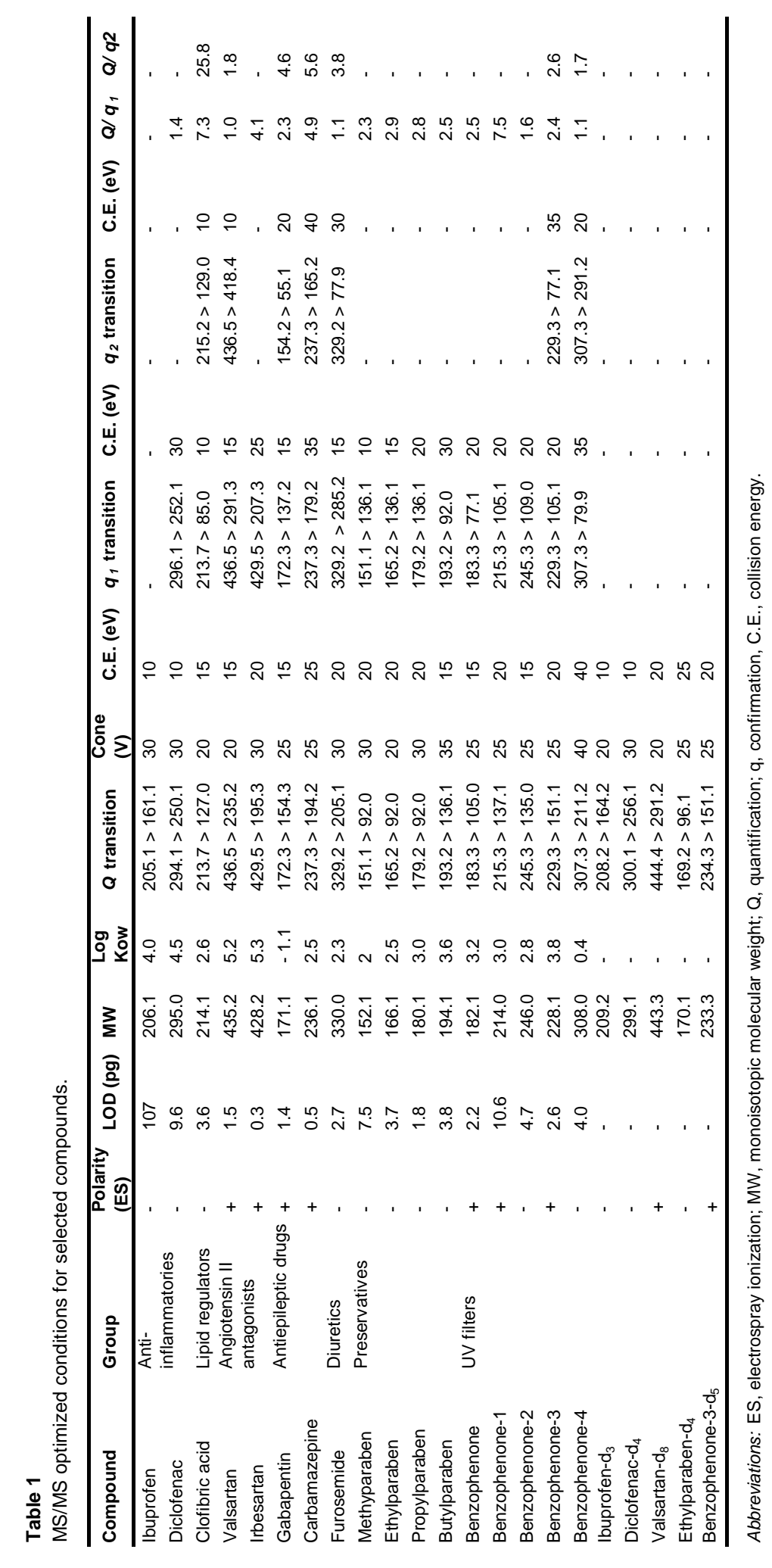




\subsection{Chromatographic conditions}

In this work, a UPLC BEH C18 column $(50 \mathrm{~mm} \times 2.1 \mathrm{~mm}, 1.7 \mu \mathrm{m})$ was chosen. To optimize the chromatographic separation, methanol and acetonitrile were evaluated as mobile phase organic solvents, and $\mathrm{HCOOH}$ at various concentrations was tested. Acetonitrile was discarded because sensitivity got worse for parabens and for most compounds ionized under positive mode.

The use of solvents without any additive provided better sensitivity for most target compounds. However, poor peak shape was observed for several compounds (e.g. valsartan, benzophenone-2), which was improved when adding $\mathrm{HCOOH}$.

The addition of $\mathrm{HCOOH}$ improved the chromatographic separation of several compounds determined in positive mode. The presence of acid also favored the chromatographic retention of acidic compounds, especially for diclofenac and clofibric acid, determined in negative mode. Finally, $0.01 \% \mathrm{HCOOH}$ as the aqueous phase, and $\mathrm{MeOH}$ as organic phase, were selected as a compromise for the simultaneous chromatographic separation of both positive and negative ionized analytes.

\subsection{Solid-phase extraction (SPE) study}

Two cartridges with different retention mechanisms were compared in this work: the hydrophilic-lipophilic balanced Oasis HLB and the mixed polymeric-cation exchange sorbent Oasis MCX. Oasis HLB was tested at natural sample $\mathrm{pH}$, while MCX cartridges required acidification of the water sample before loading in order to retain the protonated basic compounds under these conditions.

The SPE process efficiency for the selected compounds in both cartridges was tested in SW and EWW matrices. It was estimated as recovery percentage (RE).

For this purpose, the quotient between the responses obtained for samples spiked ( 0.5 $\mu \mathrm{g} / \mathrm{L}$ ) before SPE and for sample extracts spiked (50 $\mu \mathrm{g} / \mathrm{L})$ after SPE were compared [25]. This experiment was made in duplicate.

"Blank" samples, spiked only with the ILIS mix, were also processed to subtract the responses of possible target compounds that were normally present in the water samples.

The recoveries of the SPE process in both cartridges are depicted in Fig. 2 for a SW sample (Mijares river). Similar pattern was observed in EWW, although with slightly lower recoveries, surely due to the presence of matrix interferents, more abundant in wastewater than in surface water, resulting in a reduction of the sorption efficiency of cartridges. 
In the case of Oasis HLB, satisfactory RE were obtained for all compounds except for gabapentin, benzophenone and benzophenone-3, which suffered some losses in the SPE process, especially significant for gabapentin. This analyte is small, highly polar molecule, with acidic and basic groups, and it is poorly retained on Oasis HLB. On the contrary, gabapentin was efficiently retained on Oasis MCX due to protonation of the free basic amino group, yielding higher recovery (up to $90 \%$ ). In general, quantitative recoveries were observed for the majority of PPCPs in both cartridges, except for several parabens and benzophenones, which recoveries decreased considerably with $\mathrm{MCX}$. As better recoveries for a higher number of compounds were achieved with Oasis HLB, these cartridges were selected for method development and validation purposes.

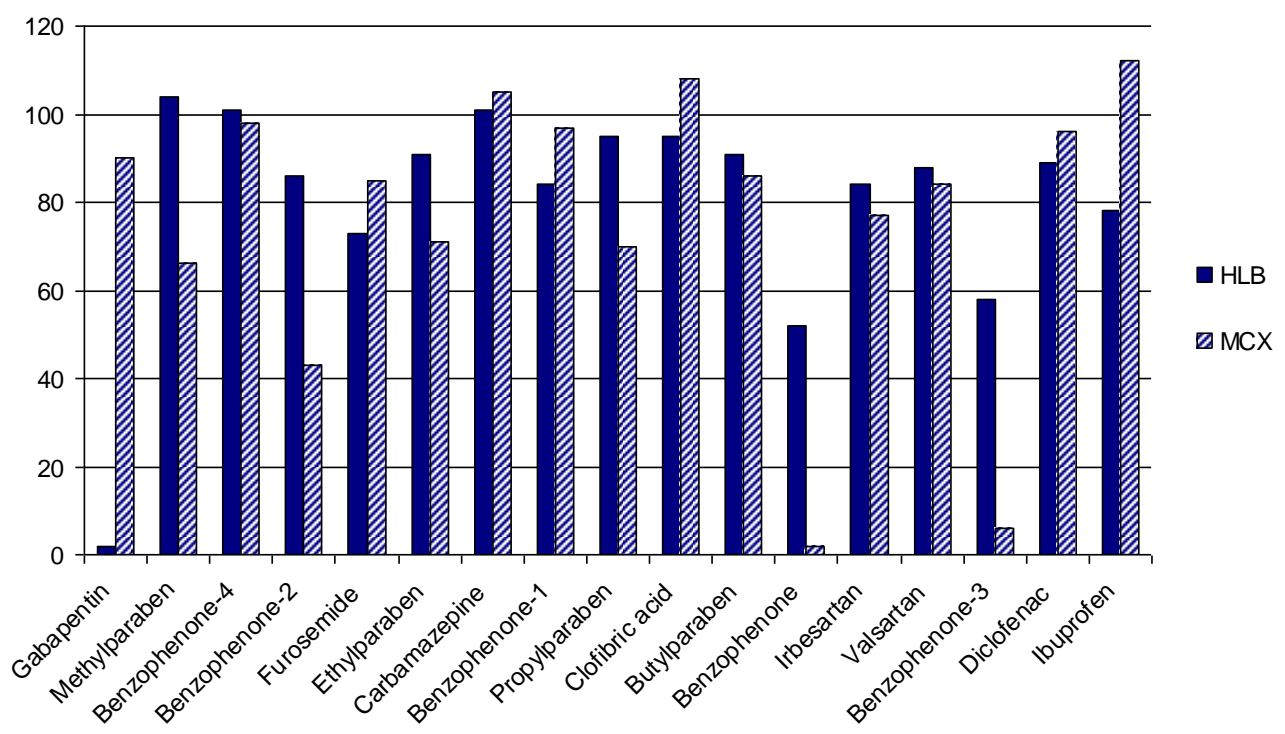

Fig. 2. Recoveries of the SPE process (RE) with Oasis HLB and Oasis MCX cartridges in SW sample.

The lower SPE efficiency for benzophenone could be corrected by the use of benzophenone-3- $d_{5}$, used as surrogate ILIS, in both SW and EWW. For the rest of compounds, although it was not strictly necessary to correct the SPE losses, the use of ILIS slightly improved their extraction process efficiency but were mostly needed for matrix effects correction, as discussed below. The only exception was gabapentin, which low recoveries could not be corrected by any of the available ILIS due to its singular physico-chemical characteristics.

\subsection{Matrix effect study}

Matrix effect is one of the main factors affecting accuracy in LC-MS/MS methods. Matrix effects are due to the presence of coeluting matrix compounds that affect analyte 
ionization leading to notable errors in quantification, unless adequately corrected or minimized. Matrix effects correction is complicated in environmental analysis, where matrix-matched calibration approach is not easy to be applied. The use of ILIS is surely the most suitable way although difficult to apply in multi-residue methods where a high number of ILIS would be required for a reliable correction. In this work, a detailed study of matrix effects was made in a notable number of water samples. To this aim, 10 different samples were chosen (five SW and five EWW) and the use of several ILIS was evaluated.

The water extracts obtained after SPE were spiked at $50 \mu \mathrm{g} / \mathrm{L}$ for each individual compound, and the ILIS mix at $25 \mu \mathrm{g} / \mathrm{L}$ was also added. For each compound, the ratio between its response in the water extract and the response of the standard in solvent at the same concentration (i.e. $50 \mu \mathrm{g} / \mathrm{L}$ ) was taken as matrix effect (ME) [25].

As expected, matrix effects in EWW were notably higher than in SW (Fig. 3) Signal suppression was observed for most PPCPs, although six of them showed ionization enhancement in both matrices (gabapentin, carbamapezine, benzophenone, benzophenone-1, valsartan and benzophenone-3). Only two compounds (benzophenone-4 and irbesartan) did not require correction in any of the samples tested. Consequently, matrix effect correction was necessary for the wide majority of PPCPs to obtain satisfactory results. In this work, the use of five ILIS was evaluated. As expected, when the analyte ILIS was available, the correction was highly satisfactory (i.e. ethylparaben, benzophenone-3, valsartan, diclofenac and ibuprofen) in all the water samples tested. For the remaining compounds, the selection of ILIS was based on the similarity in their chemical structure with the analytes under study. Thus, ethylparaben$d_{4}$ was used to correct the paraben compounds and benzophenone-3- $d_{5}$ was used for benzophenones ionized in positive mode. However, for the rest of analytes the selection of an analogue ILIS was more problematic. The best approach seemed to be the use of an ILIS with close retention time. For instance, furosemide was satisfactory corrected by ethylparaben- $d_{4}$ in SW (Fig. 3a); this ILIS was also able to compensate the ionization suppression suffered by this compound in EWW (Fig. 3b). In the case of clofibric acid, two different ILIS, both ionized under negative mode (ethylparaben- $d_{4}$ and diclofenac- $d_{4}$ ) could be selected. In SW, matrix effects correction was more satisfactory with ethylparaben- $\mathrm{d}_{4}$ but it led to undesirable enhancement when used in EWW (data not shown). On the contrary, the use of diclofenac- $\mathrm{d}_{4}$ compensated matrix effects from $50 \%$ up to around $100 \%$, so this ILIS was chosen in EWW. Similar situation was observed for carbamazepine and benzophenone-1, where benzophenone-3- $d_{5}$ was able to correct matrix effects for both compounds in SW, whilst valsartan- $d_{8}$ was found to be more suitable for their quantification in EWW.

These examples illustrate that matrix effects are both compound- and matrix-dependent and that their correction is always complicated when the analyte ILIS is unavailable. The use of the own analyte ILIS is surely the best option for satisfactory correction in all sample types, but this assumption would need to be supported by experimental data as some labelled compounds (e.g. high degree of deuterated isotopes) can have different physico-chemical behavior than the 
analyte [26]. In multi-residue methods, it uses to be unpractical to correct each compound with its own ILIS due to the high cost and the low commercial availability of ILIS reference standards. In these cases, the use of a few ILIS to compensate matrix effects for all compounds may be an alternative in the environmental field, although the satisfactory correction for all samples analyzed cannot be ensured. The choice of an appropriate internal standard is crucial in terms of compensation for matrix effects [27-29], although it seems insufficient to test it in just one given sample, due to the large variability in environmental waters and wastewaters. Thus, the study of matrix effects should be made in a notable number of samples trying to cover different situations and sample matrix compositions.

(a)

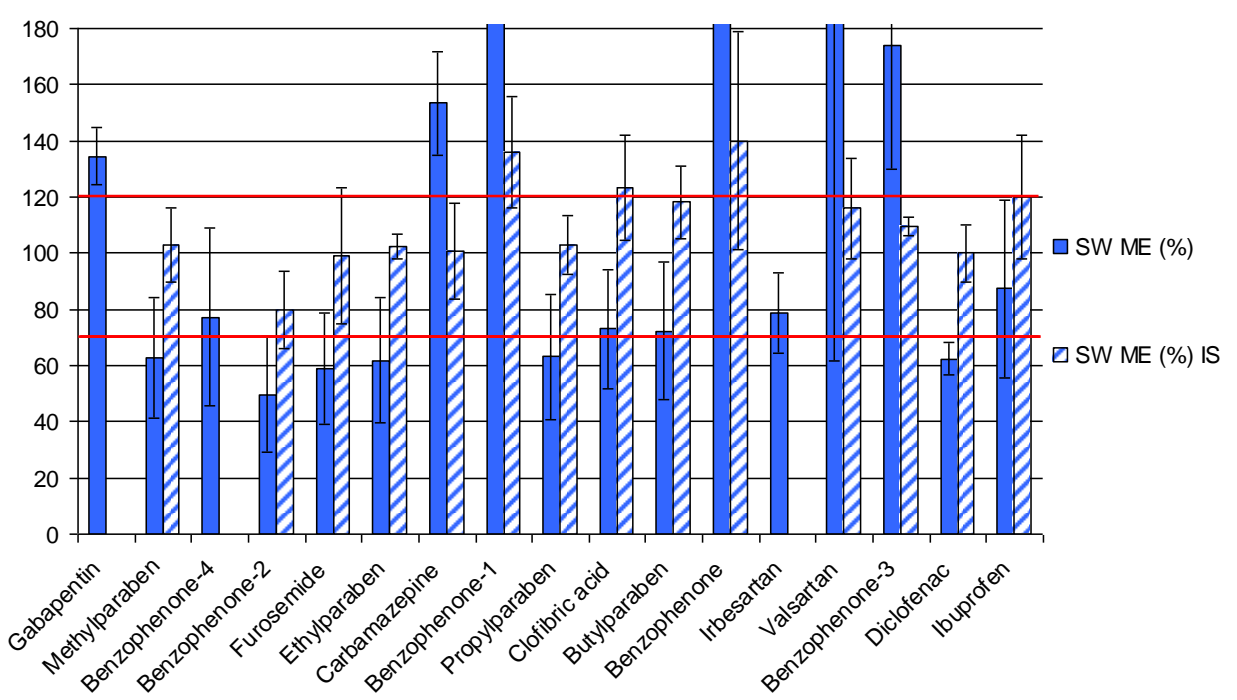

(b)

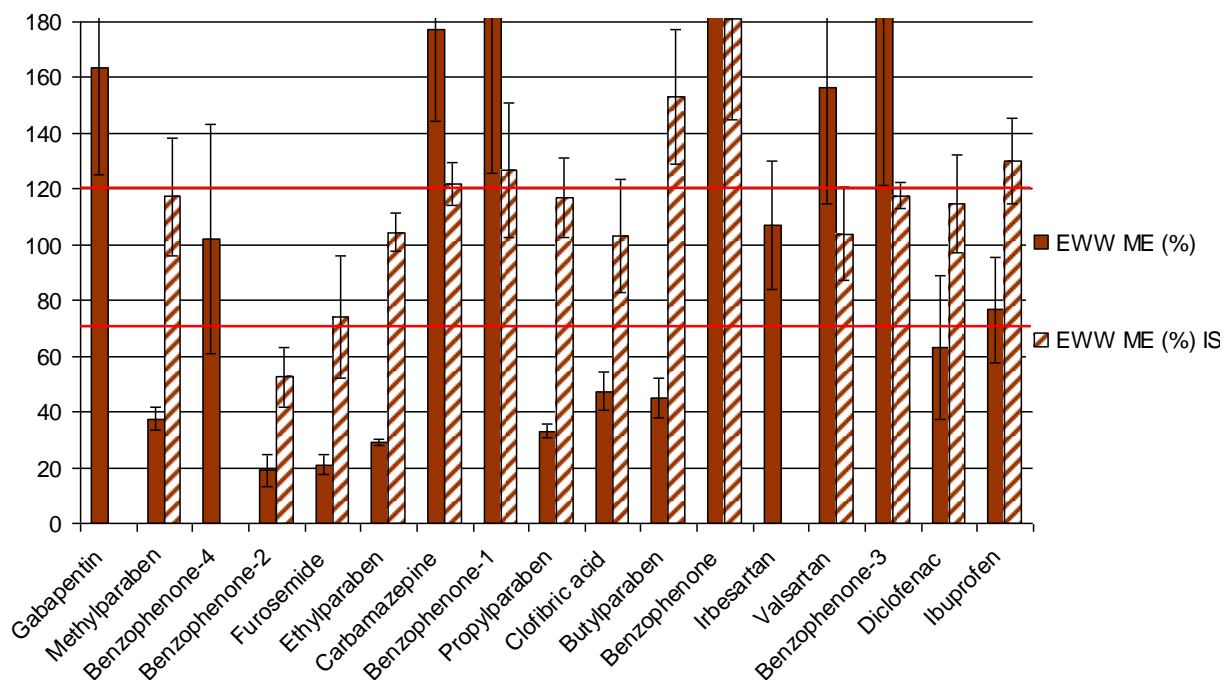

Fig. 3. Average matrix effects (ME) for (a) five different surface water samples and (b) five different effluent wastewater samples, before and after correction with ILIS. Ethylparaben-d4: used to correct for ethylparaben, methylparaben, benzophenone-2, furosemide, propylparaben, clofibric acid*, butylparaben. Benzophenone-3-d5: used for benzophenone-3, carbamazepine ${ }^{\star *}$, 
benzophenone-1**, benzophenone. Valsartan-d8: used for valsartan. Diclofenac- $d 4$ : used for diclofenac. Ibuprofen-d3: used for ibuprofen. * Correction made with diclofenac-d4 in EWW analysis. ${ }^{* *}$ Correction made with valsartan-d8 in EWW analysis.

\subsection{Method validation}

The method linearity was studied in the range $1-100 \mu \mathrm{g} / \mathrm{L}$ (these values corresponded to $0.01-1 \mu \mathrm{g} / \mathrm{L}$ in the water samples, taking into account the 100 -fold pre-concentration factor applied along the sample procedure). Calibration curves showed satisfactory correlation coefficients (greater than 0.99 ) and residuals were lower $30 \%$ for all compounds.

For validation purposes, each of the 10 water samples tested (five SW and five EWW samples) was spiked at two concentration levels. Experiments were performed in duplicate. In order to evaluate simultaneously the SPE recovery (RE) and the matrix effect $(\mathrm{ME})$, the overall process efficiency (PE) was determined. Thus, the ratio between the responses obtained for samples spiked before SPE and the response of the standard in solvent was determined [25] in the method validation.

"Blank" samples, spiked only with the ILIS mix, were also processed to subtract the responses of the target compounds that were present in the samples used for validation. PE experimental values, after correction with ILIS, for the five SW samples and the five EWW samples tested are shown inTable 2 and Table 3. ILIS correction was performed on the basis on the results obtained in the previous sections.

The method was tested at two concentration levels $(0.05$ and $0.5 \mu \mathrm{g} / \mathrm{L})$ in SW. At the lowest concentration, ibuprofen could not be validated due to the poor sensitivity. A few compounds could not be validated in all the SW samples tested due to the high analyte concentration found in some of the "blank" SW samples used in the validation (e.g. methylparaben, furosemide, propylparaben).

Recoveries in SW (calculated as PE) were satisfactory (between $70 \%$ and $120 \%$ ) for most of the compounds at the two spiking levels. Only benzophenone-4 and irbesartan were quantified without ILIS. Among the remaining compounds, five of them were corrected with their own ILIS, and the rest using an "analogue" ILIS. The use of analogue ILIS was, in general, satisfactory, but could not be always assured appropriate correction in all SW tested. For example, when carbamazepine was corrected with benzophenone-3- $d_{5}$, satisfactory recoveries were obtained except for two of the SW samples. Recoveries significantly above $100 \%$ were obtained for benzophenone-1 without ILIS correction at both spiking levels (data not shown), which was in agreement with the signal enhancement observed in the matrix effect study. The use of benzophene-3- $d_{5}$ as ILIS could not efficiently correct matrix effects, especially at the lowest level. 


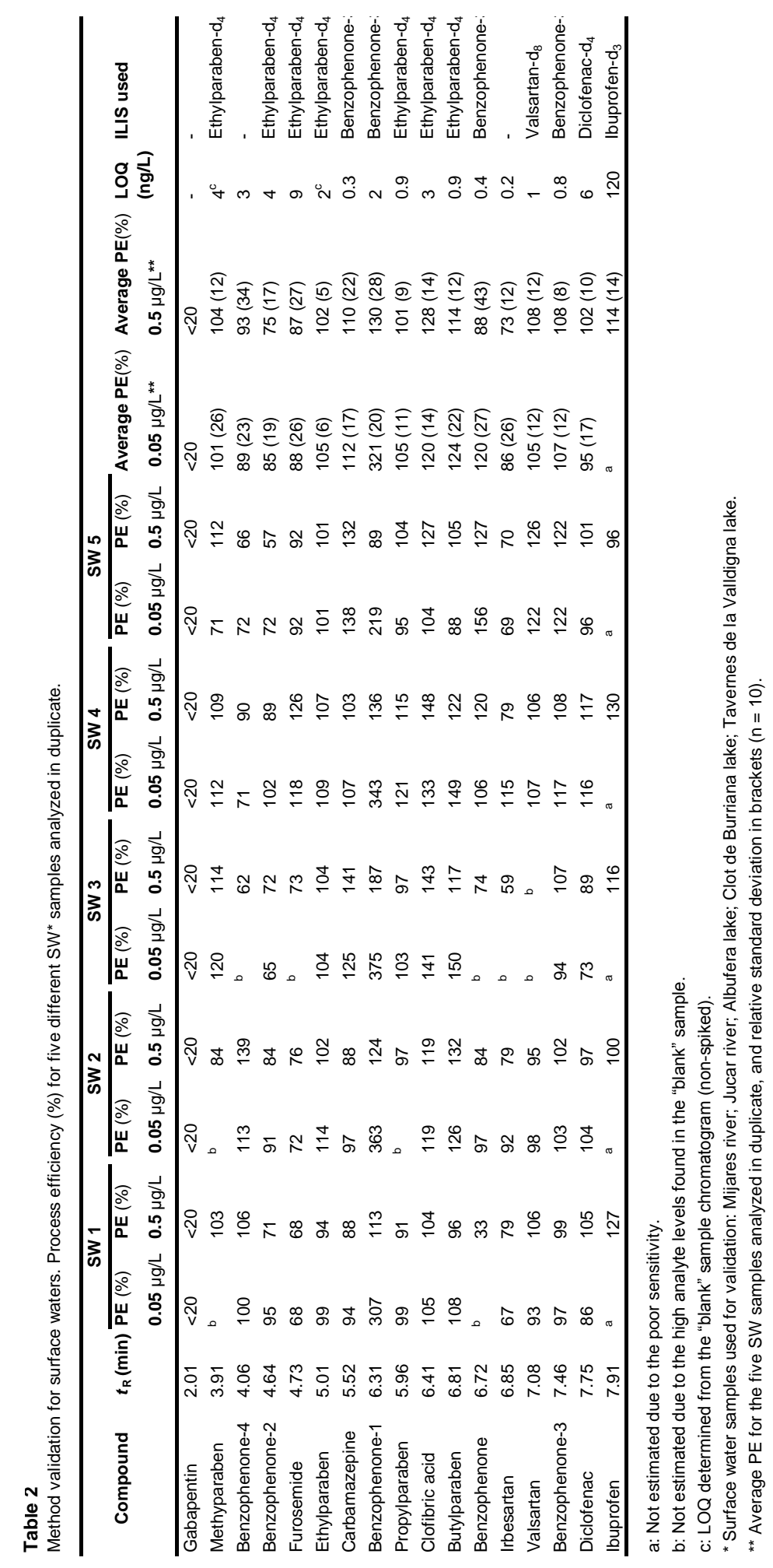




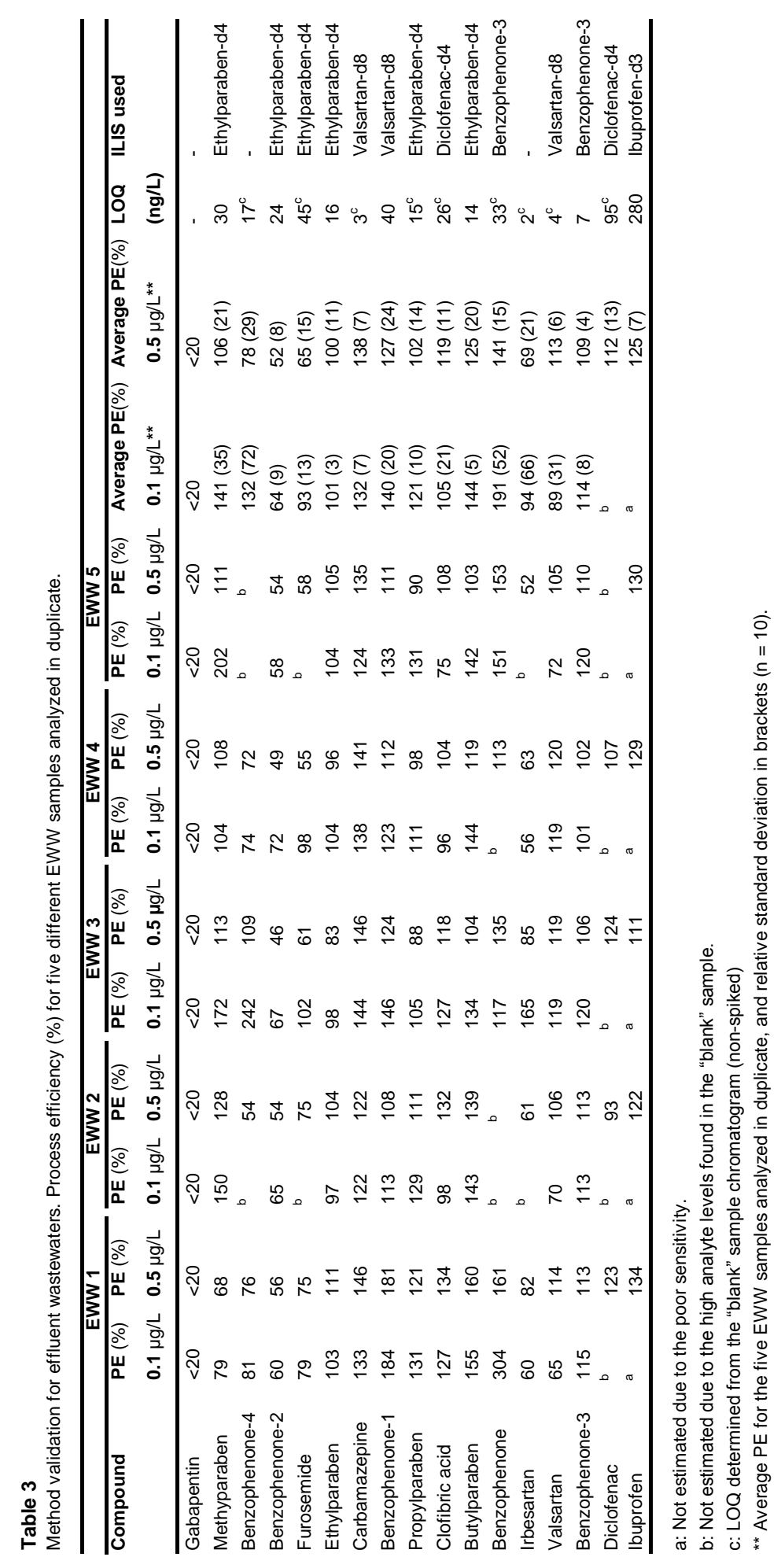


It is important to remark that, in a few cases, recoveries varied significantly from one sample to another (e.g. for furosemide, individual recoveries were in the range $68-126 \%$ at the highest level assayed). This situation was only observed when an analogue ILIS was used because the composition of aqueous samples is never the same, and unexpected matrix effects might occur.

Regarding EWW, the method was also tested in five different samples at two fortification levels each $(0.1$ and $0.5 \mu \mathrm{g} / \mathrm{L})$. All analytes were quantified using ILIS with the exception of benzophenone-4 and irbesartan, which presented acceptable recoveries without correction (see Table 3). A few compounds could not be validated in all the five samples, due to the high concentrations found in the "blank", especially in one of the samples (named as EWW 2). Recoveries were mostly satisfactory at the two levels assayed. A few compounds showed values higher than $120 \%$ in some of the EWW tested when the own analyte ILIS was unavailable (e.g. methylparaben, carbamazepine, benzophenone-1, butylparaben), illustrating that matrix effects for a sample type (e.g. effluent wastewater) do not remain constant along time and for all the samples analyzed, and can suffer notable variations. Therefore, a satisfactory correction could not be ensured for all the samples analyzed when using analogues ILIS, even though the method was tested in a notable number of water samples.

A particular case was benzophenone, as this compound presented poor extraction efficiency (RE value around 50\%) and remarkable signal enhancement (ME around 250\%), leading to a PE value near $120 \%$. Thus, the term "process efficiency" (PE) in this particular analyte might give a biased overall view of the method reliability.

Instrumental LODs are shown in Table 1 . They ranged from 0.3 to $10.6 \mathrm{pg}$, with the exception of ibuprofen. For this compound LOD was significantly higher than for the rest of analytes. The reason was its poor fragmentation, and that only a low sensitive transition could be monitored, hampering its confirmation.

Concerning LOQs, they ranged from 0.2 to $9 \mathrm{ng} / \mathrm{L}$ for SW, and were around 10 -fold higher than for the rest of analytes. The reason was its poor fragmentation, and that only a low sensitive transition could be monitored, hampering its confirmation.

\subsection{Application to environmental and waste water samples}

The method developed in this paper was applied to investigate the presence of 17 PPCPs in different types of water: reservoir water (Colombia), and SW and EWW (Spain).

In every sequence of analysis, the calibration curve was injected twice, at the beginning and the end of the sample batch. Moreover, quality control samples (QCs) were included in every sample sequence. QCs consisted on SW, EWW or reservoir water spiked at $0.5 \mu \mathrm{g} / \mathrm{L}$. 
They were prepared randomly selecting one of the water samples analyzed within the batch, and were analyzed following the same analytical procedure than samples. QC recoveries in the range $60-120 \%$ were considered as satisfactory (Tables 4 and Table 5).

Confirmation of positive findings was carried out by calculating the peak area ratios between the quantification $(Q)$ and confirmation $\left(q_{1}\right.$ and $\left.q_{2}\right)$ transitions. The finding was considered as true positive when the experimental ion-ratio was within the tolerance range [30], and the retention time in the sample within $\pm 2.5 \%$ the retention time, when compared with a reference standard.

A great variety of PPCPs were detected in a wide range of concentrations in samples from Spain (Table 4). In SW, 14 out of 17 PPCPs were detected at least once. Among them, irbesartan, benzophenone, benzophenone-3, methylparaben and propylparaben were quantified in all SW samples. On the contrary, clofibric acid, benzophenone-2 and butylparaben were not detected in any of the samples. The highest concentrations found were for valsartan (up to $6.3 \mu \mathrm{g} / \mathrm{L}$ ). These results are in fairly good agreement with data reported by other authors. For instance, in a study carried out by Kasprzyk-Hordern et al. [31], the pharmaceuticals ibuprofen, diclofenac, gabapentin, furosemide and valsartan were also frequently found in SW samples from United Kingdom and their concentration levels were similar to those found in our study. Regarding PCPs, in other works highest concentrations were found for methylparaben and benzophenone-4 $[10,31]$, which is in accordance with the present study. The presence of pharmaceuticals in "Albufera de Valencia" lake has been previously reported in literature [32], concretely carbamazepine (2.2-31 ng/L), ibuprofen (n.d.-3913 ng/L), clofibric acid (n.d. $-71.4 \mathrm{ng} / \mathrm{L}$ ) and diclofenac (n.d. $-260.9 \mathrm{ng} / \mathrm{L}$ ), among other compounds. These data are in the line of the present work, where carbamazepine $(12 \mathrm{ng} / \mathrm{L})$ and ibuprofen $(<\mathrm{LOQ})$ were also found in the same area. The most polluted source was Jucar river (in Valencia) where 13 out of 17 targeted PPCPs were detected, the majority of them at the highest concentration levels found in this work. 


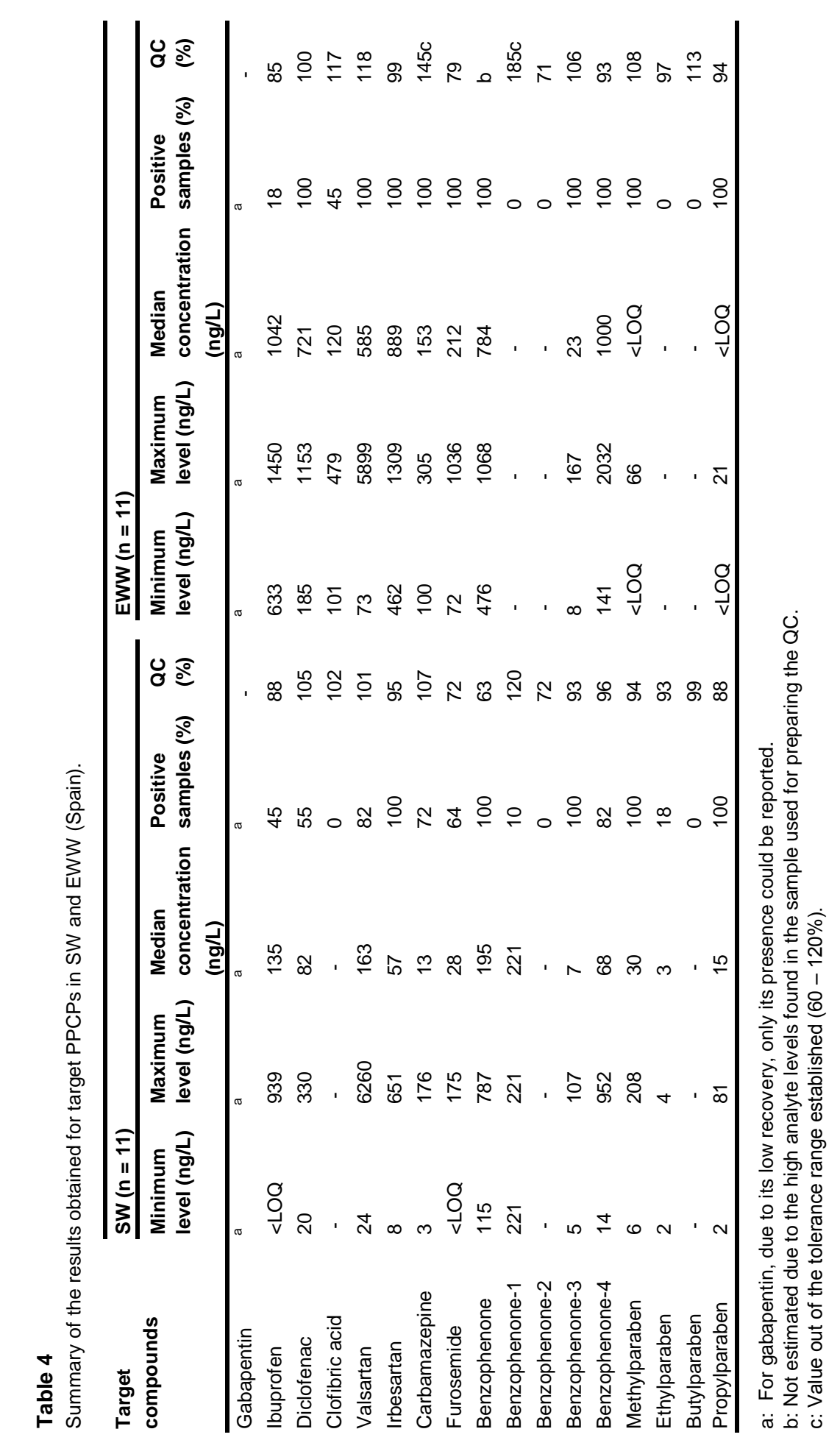


Regarding EWW, 10 of the target PPCPs were detected in all the samples (benzophenone, benzophenone-3, benzophenone-4, methylparaben, propylparaben valsartan, irbesartan, diclofenac, carbamazepine and furosemide). On the contrary, four compounds were not detected in any EWW sample: benzophenone-1, benzophenone-2, ethylparaben and butylparaben, which was in general agreement with data obtained in SW (benzophenone-1 and ethylparaben were found in only one and two surface waters, respectively). The highest levels were found for benzophenone-4 and valsartan, which were in the range 0.14-2 and 0.07$5.9 \mu \mathrm{g} / \mathrm{L}$, respectively. Valsartan was by far the compound present at higher levels in both SW and EWW samples. As expected, concentrations in EWW were usually higher than in SW. In agreement with other studies [10] and [31], preservatives were found at very low levels. This was also observed for benzophenone-1, benzophene-2 and benzophenone-3, whilst benzophenone-4 was one of the compounds found at higher concentrations.

For gabapentin, due to its low recovery, only qualitative data were obtained. This compound was detected in four SW and in all EWW samples analyzed. It was confirmed by the presence of chromatographic peak at the three SRM transitions acquired for this compound.

In relation to reservoir water from Colombia, both water sources showed less contamination by PPCPs than surface water from Spain. In fact, there were no evidences on the presence of gabapentin, ibuprofen, diclofenac, clofibric acid, valsartan, irbesartan, carbamazepine, furosemide and benzophenone-2 in any reservoir. A summary of the results obtained is shown in Table 5. On the contrary, the occurrence of parabens and two benzophenones was highly relevant in both reservoirs. In reservoir 1, methylparaben and propylparaben were present in all samples in a wide range of concentrations. These compounds were also found in most of the samples of the reservoir 2 (93\% of positive samples). This is in accordance with previous studies where methylparaben was the preservative most frequently detected in river waters from the United Kingdom [31]. Ethylparaben was found in around $70 \%$ of samples from reservoir 1 and in all samples from reservoir 2, although at very low levels, commonly below $10 \mathrm{ng} / \mathrm{L}$. Benzophenone and benzophenone-3 were detected in both reservoirs in a notable number of samples. On the contrary, benzophenone-1 and benzophenone-2 were only detected in the reservoir 1 in just one sample. 


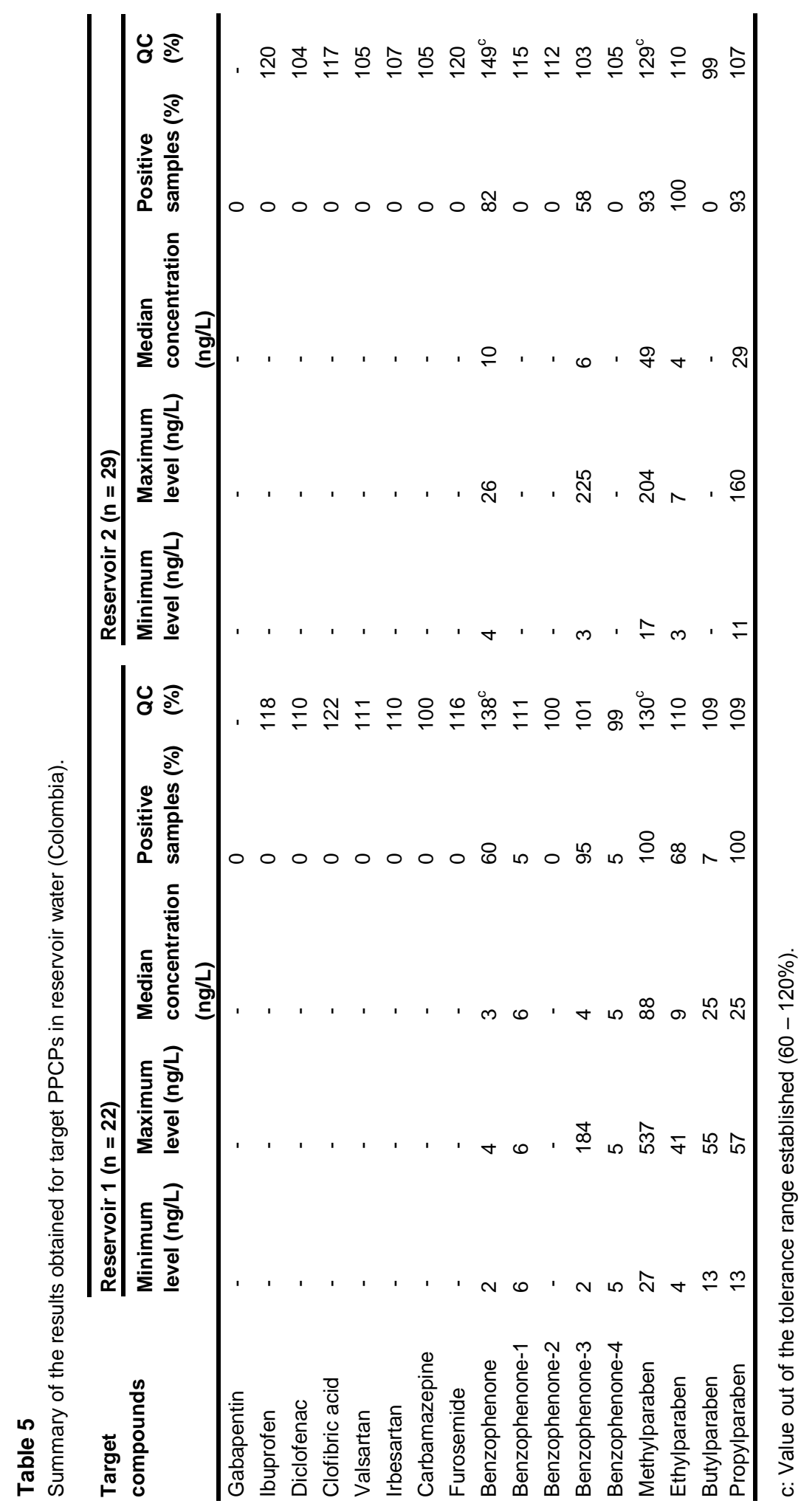


Recoveries for the Quality Control samples (QCs) included in every sequence of analysis were, in general, satisfactory, within a range of $60-120 \%$ (see Table 4 and Table 5). Only a few exceptions were observed in EWW and in the reservoir water samples. In all these cases, QCs recoveries were higher than $120 \%$ and corresponded to analytes that were corrected with an analogue ILIS. A correction factor was applied when the compound was detected and quantified in the samples (five out of six cases).

As an illustrative example, Fig. 4 shows a positive finding of methylparaben, propylparaben, benzophenone and benzophenone-3 in one of the water samples collected at reservoir 2. From the data obtained on PCPs in the water from reservoirs, we did not observe specific trends in the concentrations of these compounds at the different depths considered in the water column.

a

\section{b}

C

d
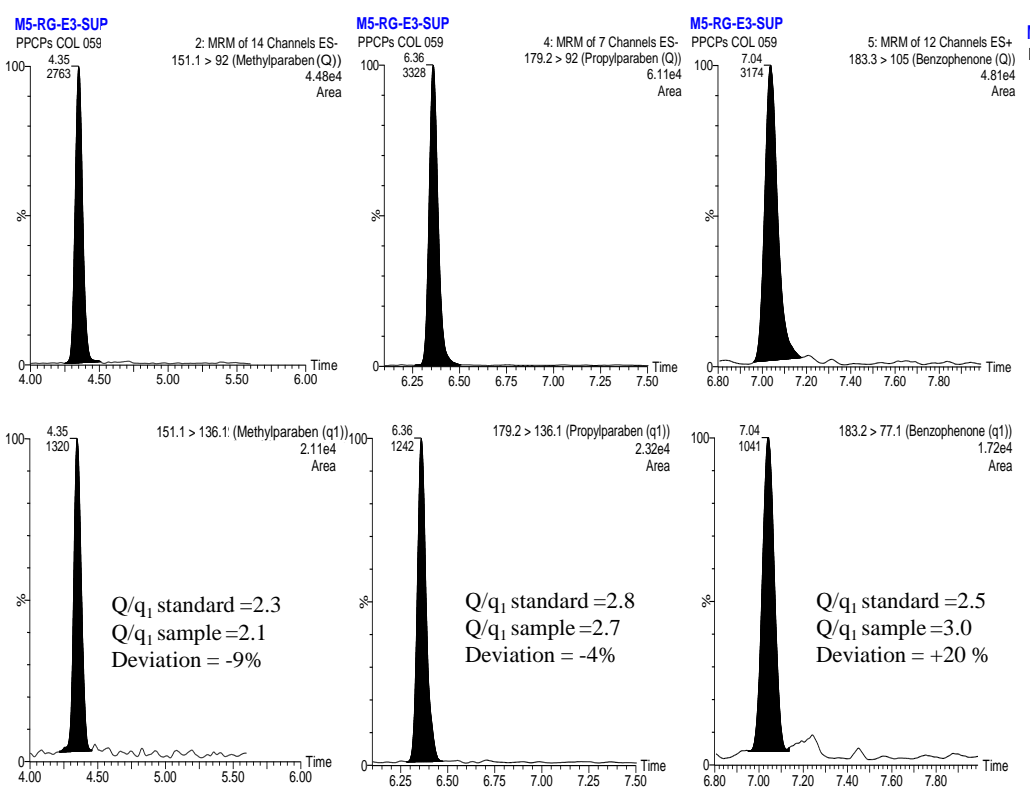

M5-RG-E3-SUP
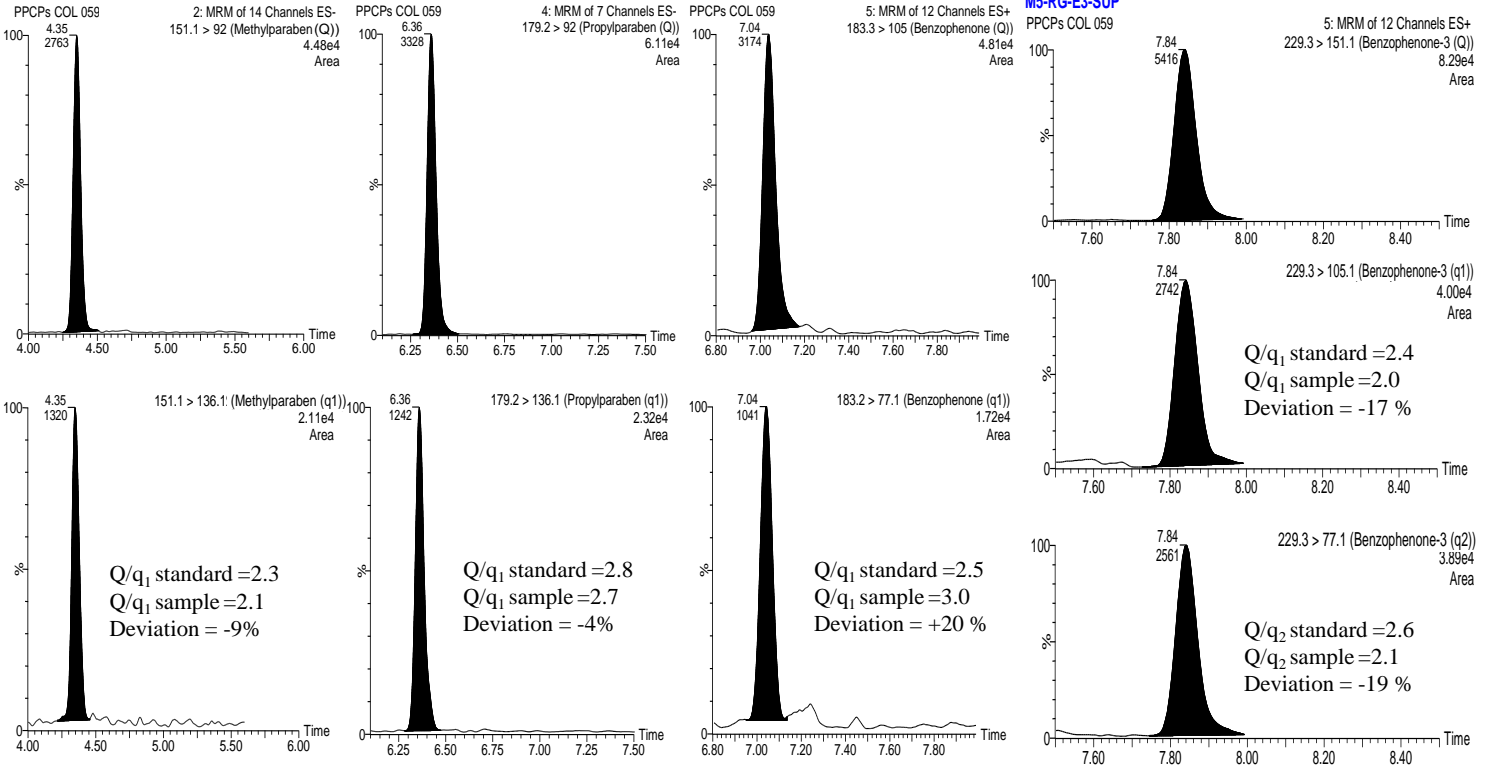

Fig. 4. UHPLC-MS/MS chromatograms for selected analytes for a reservoir water sample. Methylparaben (141 ng/L), propylparaben (142 ng/L), benzophenone $(7 \mu \mathrm{g} / \mathrm{L})$, benzophenone-3 (6 ng/L).

None of the target pharmaceuticals was detected in the reservoir waters from Colombia. This was unexpected, especially for diclofenac and ibuprofen, which seem to be widely consumed in this country. The reason might be found in the high dilution taking place due to the large capacity of these reservoirs. 
The influence of human activities in areas near the Colombian reservoirs seems clear in the case of PCPs (for example, reservoir 1 is used as a recreation place and it is surrounded by residences), but more research and data would be required to understand the fate and behavior of pharmaceuticals on this aquatic environment.

The method developed will be implemented in the laboratory of GDCON, University of Antioquia, from 2012. Thus, it is expected that more data will be available on PPCPs in the aquatic environment of this area in the near future.

\section{Conclusions}

In this paper a multi-residue method for the simultaneous quantification and confirmation of 17 emerging contaminants, including widely used pharmaceuticals and personal care products, has been developed and applied to surface water samples and effluent wastewater from Spain and Colombia.

The method has been evaluated and validated in a total of 10 water samples (five surface waters and five effluent wastewaters). This number of samples, higher than usual in most method validations reported for PPCPs, was chosen in order to have a wider overview of the method performance in different water samples. The matrix effects study performed in this work shows severe signal suppression/enhancement, especially in some of the EWW tested. The correction with analyte ILIS was satisfactory in all sample types. The use of analogues ILIS was rather satisfactory, although it could not ensure appropriate correction in all the analyte/water samples combinations, as matrix effects are both compound and matrix dependent. As well known, the composition of water samples is never the same and notable changes can occur along the time and as a function of their origin. As a consequence, matrix effects that commonly affect LC-MS/MS methods can be rather different from one sample to other. Thus, the selection of appropriate analogue ILIS, when the analyte ILIS is not available, is controversial and should be only made after a careful study on matrix effects in a considerable number of water samples of different types and origin.

The results obtained in this work after the application of the method to 73 water samples (surface: rivers, lakes and reservoirs, and effluent wastewater) showed the wide majority of the target analytes were present in the samples analyzed. The importance of including personal care products, which have received less attention than pharmaceuticals during the last years, is evidenced by the widespread occurrence of these compounds in the samples under study. To the best of our knowledge, the presence and distribution of PPCPs in Colombia have not been reported, and this is the first publication providing data on PPCPs in this country. 


\section{Acknowkedgments}

This work has been developed under financial support of the Ministry of Education and Science, Research Project CTQ-2009-12347.

The authors are very grateful to FACSA for providing wastewater samples used for the method validation. M. Martínez is especially grateful to "Enlazamundos" Programme (Colombia) for her financial support and to Carolina Aristizábal for providing reservoir water samples from Colombia and her support to perform this work.

The LC-MS/MS instrument (Waters, TQD) from Serveis Centrals d'Instrumentació Científica (SCIC) of University Jaume I has been used in this work. The authors acknowledge the financial support of Generalitat Valenciana as research group of excellence PROMETEO/2009/054. E. Gracia-Lor is very pleased to University Jaume I for her pre-doctoral grant.

\section{References}

[1] J.B. Ellis, Environ. Pollut. 144 (2006) 184-189.

[2] C. Hao, X. Zhao, P. Yang, Trends Anal. Chem. 26 (2007) 569-580.

[3] E. Walters, K. McClellan, R.U. Halden, Water Res. 44 (2010) 6011-6020.

[4] C.I. Kosma, D.A. Lambropouloub, T.A. Albanisa, J. Hazard. Mater.179 (2010) 804-817.

[5] M. Gros, M. Petrovic, D. Barceló, Talanta 70 (2006) 678-690.

[6] W. W. Buchberger, J. Chromatogr. A 1218 (2011) 603-618.

[7] C. Wang, H. Shi, C. D. Adam, S. Gamagedara, I. Stayton, T. Timmons, Y. Ma, Water Res. 45 (2011) 1818-1828.

[8] D.W. Kolpin, E.T. Furlong, M.T. Meyer, E. M. Thurman, S.D. Zaugg, L.B. Barber, H.T. Buxton, Environ. Sci. Technol. 2002, 36, 1202-1211.

[9] Z. Moldovan, Chemosphere 64 (2006) 1808-1817.

[10] M. Pedrouzo, F. Borrull, R.M. Marcé, E. Pocurull, Trends Anal. Chem. 30 (2011) 749-760.

[11] K. Fent, A. Zenker, M. Rapp, Environ. Pollut. 158 (2010) 1817-1824.

[12] H-K. Jeon, Y. Chung, J-C. Ryu, J. Chromatogr. A 1131 (2006) 192-202.

[13] G. J. Nohynek, H. Schaefer Regul. Toxicol. Pharm. 33 (2001) 285-299.

[14] A. Salvador, A. Chisvert, Anal. Chim. Acta 537 (2005) 1-14. 
[15] [15] W. Li, Y. Ma, C. Guo, W. Hu, K. Liu, Y. Wang, T. Zhu, Water Res. 41 (2007) 35063512.

[16] Jiménez-Díaz, F. Vela-Soria, A. Zafra-Gómez, A. Navalón, O. Ballesteros, N. Navea, M.F. Fernández, N. Olea, J.L. Vílchez, Talanta 84 (2011) 702-709.

[17] E. Eriksson, H.R. Andersen, A. Ledin, J. Hazard. Mater., 156 (2008) 240-259.

[18] J. Regueiro, E. Becerril, C. Garcia-Jares, M. Llompart, J. Chromatogr. A 1216 (2009) 4693-4702.

[19] D. Fatta-Kassinos, S. Meric, A. Nikolaou, Anal. Bioanal. Chem. (2011) 399:251-275.

[20] E. Gracia-Lor, J.V. Sancho, F. Hernández, J. Chromatogr. A 1217 (2010) 622-632.

[21] F. Hernández, M. Ibáñez, E. Gracia-Lor, J.V. Sancho, J. Sep. Sci. 34 (2011) 3517-3526.

[22] B. Kasprzyk-Hordern, R.M. Dinsdale, .J. Guwy, Anal. Bioanal. Chem. 391 (2008) 12931308.

[23] González-Mariño, J.B. Quintana, I. Rodríguez, R. Cela, Rapid Commun. Mass Spectrom. 23 (2009) 1756-1766.

[24] M. Pedrouzo, F. Borrull, R.M. Marcé, E. Pocurull, J. Chromatogr. A 1216 (2009) 69947000 .

[25] B.K. Matuszewski, M.L. Constanzer, C.M. Chavez-Eng,. Anal. Chem. 75 (2003) 30193030.

[26] C. Ripollés, J.V Sancho, F.J. López, F. Hernández, J. Chromatogr. A 1243 (2012) 53-61.

[27] B. Kasprzyk-Hordern, R.M. Dinsdale, A.J. Guwy, Talanta, 74 (2008) 1299-1312.

[28] E. Gracia-Lor, J.V. Sancho, F. Hernández, J. Chromatogr. A 1218 (2011) 2264-2275.

[29] J.M. Marín, E. Gracia-Lor, J.V. Sancho, F.J. López, F. Hernández, J. Chromatogr. A 1216 (2009) 1410-1420.

[30] European Union Decision 2002/657/EC, Off. J. Eur. Commun., L221 (12 Aug. 2002), 8-36.

[31] B. Kasprzyk-Hordern, R.M. Dinsdale, A.J. Guwy, Water Res. 43 (2009) 363-380.

[32] P. Vázquez-Roig, V. Andreu, M. Onghena, C. Blasco, Y, Picó, Anal. Bioanal. Chem. 400 (2011) 1287-1301. 